\title{
Linfosarcomas del abdomen en niños
}

De las lesiones tumorales del niño mís de la mitad la constituye los tumores sólidos, de los cuales el linfosarcoma es para nosotros el más frecuente.

\section{Tabla l}

Hospital Roberto del Rio 1960.1975

\begin{tabular}{|c|c|c|}
\hline I.eucctnias & 180 & casos \\
\hline Hodgkin & 49 & $"$ \\
\hline I.infollas no Hodgkin & 57 & $"$ \\
\hline Nouroblastona & 11 & $"$ \\
\hline Sarcomas de partes bI. & 9 & $"$ \\
\hline T'xmores det ovario & 29 & $*$ \\
\hline Tumores del testiculo & 17 & 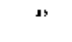 \\
\hline Tumores de los hueses & 10 & $"$ \\
\hline 'Гutuoren Hepáticos & b & $"$ \\
\hline Tumores sicl SNC: & 4 & 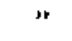 \\
\hline Otros & I8 & + \\
\hline Total & 402 & casos \\
\hline
\end{tabular}

Si bien en la Enfermedad de Hodgkin el cnforue diagnistico y terapeutico clel adulu es aplicable al nif̂́o, no sucedle lo mismo con el I,infosarcoma, que es cle evolución mucho mís rípida y requiere un watamiento distinto y más agresivo. Se lcucomiza con mityor facilidul y liene menos espectativas de vida. $(5$. (6).

Fn los úlrimos años se ha logrado una mejoría substancial en cl pronóstico de estos tumores, gracias a un tratamiento múltiple con

\footnotetext{
- Servicio de Cirugia. Hospital Roberto del Río.

* Departamento de Pediacria, Hospital Rulerto del Rín.

* "Servicio de Anatomia Patológica, Hospjtal Roberto del Rio.
}

Rev. Chilena de Pedivitría, Vol. 47, No ], 1976

\author{
Drs, Guntavo Aldunate N** Eugenia Emparanza*", \\ David Mirkin W.
}

varias drogas combinadas, cirugia amplia, radioterapia e inmunoterapjia. (8) .

Fil reconocimiento más preciso del tipo histológico del linfosarcoma y su grado de invasión o etapa han permitido adccuar la terapia hasta alcanzar cifras sorprendentes de curación, de 60 a $80 \%$ (4).

Destle que Burkitt en 1958 describiera un Linfoma maligno con distribución geográfica en Africa Tropical y clestacara su clínica e histologit projiass, se han comunicado cada vez mis casos de cste tipo de tumor, incluso en nuestro país. Es importante identificar este Iinfoma, ya que tiene un alto ínclice de curabilititul con la Quimioterapia (3-7-9).

\section{MATERIAL}

En muestra serie la localización inicial del tumor corresponde casi en la mitad de Ios casos al abdomen, luego al mediastino y ganglios periféricos. En 6 casos cra generalizado y no se pudo prerisar de chóncle partió. Puede apre. ciarse también que los linfosarcomas abdominales sc leucemizan muy raramente.

Tabla 2

Localizacion originarin $N^{o}$ de ansos Ieucemización

\begin{tabular}{lrrr}
\hline Abdomen & 27 & 1 & $5 \%$ \\
Merliastino & 12 & 6 & $50 \%$ \\
Ganglios periféricos & 7 & 4 & $57 \%$ \\
Cenutralizados & 6 & 6 & $100 \%$ \\
Otros sitios & 5 & 4 & $80 \%$ \\
\hline Total & 57 & 21 & $\mathbf{3 7 \%}$ \\
\hline
\end{tabular}


En la distribución por sexo hay un franco predominio de los varones, que es más evidente en los casos de localizacion abdominal (figu. ra No 1). En la distribución por edad se puede
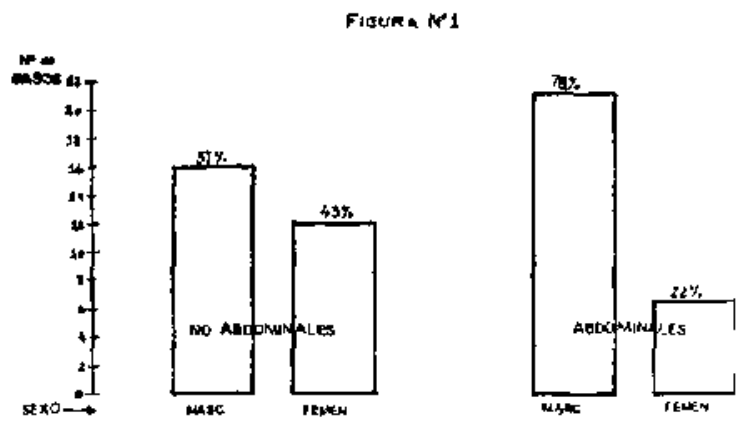

observar que las formas abdominales tienden a presentarse en pacientes de menor edad, imitando un poco la curva de distribución de la Leucemia. En cambio, los extraabdominales pueden verse con mayor frecuencia en la edad escolar (figura No 2).
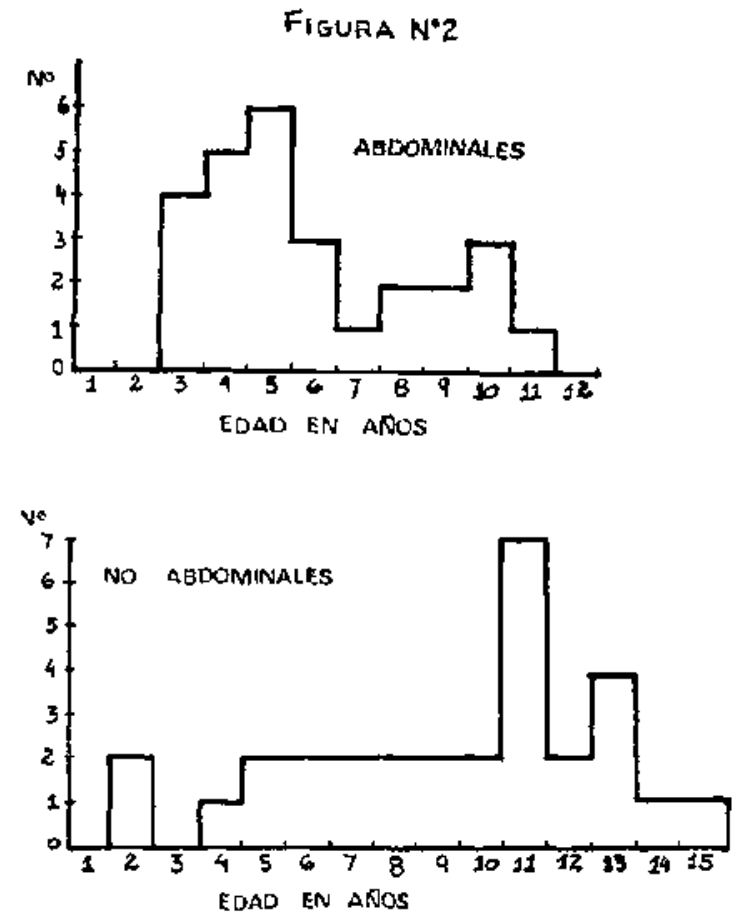

\section{RESULTADOS}

Limitándonos al análísis de los 27 linfosarcomas abdominales debemos señalar que no siempre es posible en la exploración quirúrgica del abdomen poder precisar cuál es la locali. zación inicial del tumor: 10 pacientes presentaban una invasión difusa de mesos, intentino y pared posterior del abdomen, con líquido hemorrágico abundante en el peritoneo. En ellos sólo pudo tomarse una biopsia que confirmó el Linfoma. En otros nin̄os más afortunados, la lesión produjo una obstrucción intestinal por compresión o invaginación, lo que permitió un diagnóstico más precoz y se pudo hacer una resección intestinal arnplia.

Tabla $\mathbf{3}$

\section{SITIO DE ORIGEN EN EL ABDOMEN}

\begin{tabular}{lrc}
\hline Yeyuno & l caso \\
Ilcon & 7 & $"$ \\
lleocecal & 4 & $"$ \\
Colon & 5 & $"$ \\
No precisable & 10 & $"$ \\
\hline Tota! & 27 & casos \\
\hline
\end{tabular}

El motivo de consulta suele ser en los niños más pequeños, el compromiso del estado general y la distensión del abclomen. In los niños mayores es el dolor abdominal impreciso, cuya causa no se logra aclarar hasta que la pilpación de una o varias masas abdominales pone en la pista del diagnóstico.

En la Figura No 3 hemos separado tres gru-

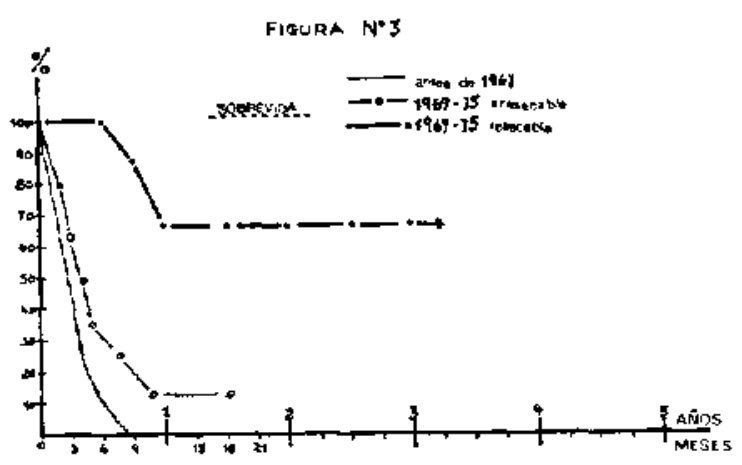

pos de pacientes: 11 niños operados antes de 1966, cuando aún no disponíanos de quimioterapia eficaz. Todos fallecieron antes de 6 meses (1). Exporiencia similar publicaron Gu. tiérrez y cols. en 1968 (2).

Desde 1968 funciona en nuestro Hospital 
In Comité de Tumores integrado por Hematólogos, Cirujanos, Anatomopatólogo y Radioterapeutas que fijan el programa de tratamiento y siguen la evolución de los casos. Descle 1969 a 1975 se han controlado 16 niños con linfosarcoma abdominal con dos grupos bien caracteristicos:

a) 8 nin̄os en los que no fue posible efertuar resección debido a la gran invasión tumoral del abriomen. Sólo uno de ellos sobrevive al año de operado y la biopsia mostró rasgos histológicos sugerentes de Linfoma de Burkitt.

b) 8 niños en los que se pudo efectuar una resección amplia del intestino y mesos infiltrados. 3 de ellos fallecieron antes de un año, uno por manifestaciones tóxicas a la Quimioterapia y dos por recidiva clel tumor. De los 5 que sobreviven. 3 han sobrepasado los 5 años de control, otro va para los cuatro años y el quinto recién lleva un año y medio. (Fig. 4).

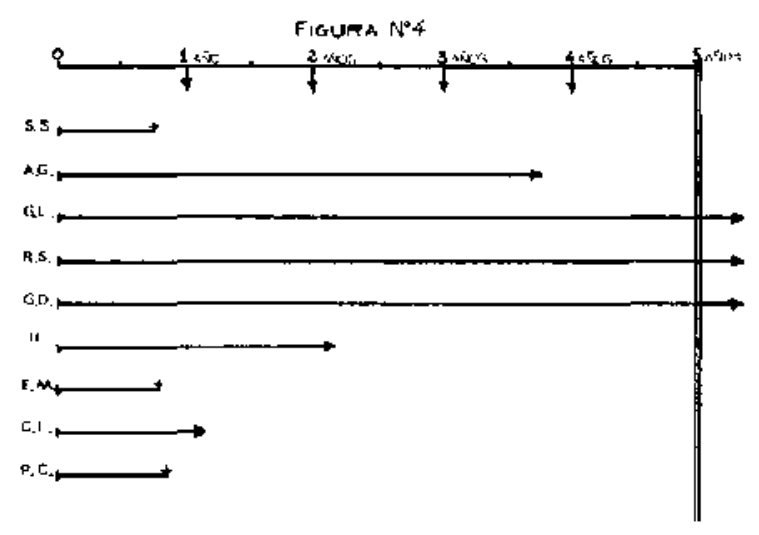

Digno de destacar es el caso de F. G. D. G. Operado en 1969 por una invaginación intestinal con Linfoma ileocecal. Se le practicó una resección del íleon terminal y cecoascendente. Siguió con quimioterapia cíclica combinada con Prednisona, Vincristina y Endoxan por 2 semanas con tres de descanso. Cuatro años después, en 1973 hizo una segunda obstrucción a $80 \mathrm{~cm}$., la anastomosis anterior, en el ileon. Ha cumplida 3 años de esta segunda operación y no hay signos de recidiva. Pero ha presentado hematuria por efectos secundario del Endoxan.

La radioterapia se lia usado en muy pocos casos, en los más antiguos, ya que en nuestra experiencia no ha sido efectiva en prolongar la sobrevida y agrava las complicaciones' de la quimioterapia.

\section{CONCLUSIONES}

Podemos decir que la sobrevida de los niños con linfosarcoma abdominal ha mejorado gracias a la integración de los distintos especia. listas en un Comité de Tumores que establece un diagnóstico más exacto y combina los recursos más adecuados para cada caso, se preocupa del cumplimiento del programa terapéutico y controla periódicamente estos pacientes por largos años. En la experiencia presente, los hechos más destacables son los siguientes:

I. El linfosarcoma es en nuestro medio el tumor sólido más frecuente en los niños.

2. Su localización abdominal es la más frecuente.

3. La Ieucemización en las formas abdominales es muy rara de ver.

4. Antes de 1966 la mortalidad era de $100 \%$.

5. Desde el uso de quimioterápicos en 1968 se ha logrado una sobrevida de $31 \%$. En Ios casos que se logra una resección quirúrgica de las lesiones la sobrevida llega a un $62 \%$.

\section{RESUMEN}

Se analiza Ia sobrevida de 27 casos de Linfosarcomas abdominales en niños atendidos en el Hospital Roberto del Río desde 1960 a 1975. Cuando se logra la resección quirúrgica amplia de las lesiones y se dispone de quimioterápicos eficaces puede experarse una sobrevida superior al $60 \%$.

\section{SUMMARY}

27 cases of abdominal lymphosarcomas in chil. dren were seen at Hospital Roberto del Rio in Santiago from 1960 to 1975.

When abdominal resection is feasible and chemotherapeutic drugs are given for a long period, cure rate is over $60 \%$ in our patients. 
and the non-Hodgkin's lymphomas in childhood". Scm. Oncology 1: 1974.

1. Goepfert. H., "Linfoma intestinal en la infancia" Arch. Soc. Cir. Chile 22: 381, 1970.

2, Gutiérrez $C_{*}$, Veloso A., Salonón A., Balaz O., "I.infosarcoma abdominal en el nifno". Rev, Chil. Pediat. 39: 797,1968 .

3. Hurtado, Rodrigo y Cols., "Linfoma de Burkitt". Pediatria 14: 5.10, 1971 .

4. Murphy M., Lois, "The multidiscipline team in a cancer center". Cancer \$5: 876, 1975.

5. Murphy Sh, B., David L., W., "Hodgkin's disease

6. Pochedly, Carl., "The Lymphosarcomas in mayor problems in childhood cancer". Edit. Ch-Thomas. Illinois, 1973 .

7. Rivera G., Rajeric J.. J\}az M., del Pozo H., Jel Rio A., "Tumor de Burkitt", Rev. C:Iil, de Pediat. 10: 2 A I, 1969 .

8. Sulliran. Margaret P., "Treatment of Iymphoma", Cancer 35: 991, 1975.

9. Ziegler J. L.. "Management of Burkitt Lỵmphoma". Ped. Annals 4: 99, 1975. 